\title{
Post-Implementation Cost-Analysis of the ICD-10-CM Transition on Small and Medium-Sized Medical Practices
}

\section{Parth Desai* and Rachelle Eljazzar}

MS-4, Mercer University School of Medicine, Macon, Georgia, USA

\section{Abstract}

Introduction: On October 1, 2015, the United States made a major transition in its medical billing and coding system, by switching from ICD-9-CM to ICD-10-CM. Several cost-analysis studies have attempted to estimate the eventual impact of the ICD-10-CM transition on medical practices, but all were completed prior to the actual transition deadline. Our study seeks to assess the post-implementation financial impact of the transition on small and medium medical practices which used a set of non-profit resources for their implementation.

Methods: 6,000 medical practices were randomly selected from the approximately 70,000 user database of a non-profit ICD-10 provider and emailed a seven question survey. 419 practices completed the full survey (8.5\% response rate), providing practice demographics, as well as estimates for the hours spent and cost accrued on the implementation.

Results: Based on the reported data, the average total explicit cost of the ICD$10-\mathrm{CM}$ implementation was $\$ 1,206$ for small medical practices and $\$ 2,462$ for medium medical practices. The average total number of staff hours spent was 61.2 hours for small practices and 139 hours for medium-sized practices. The average total number of physician hours spent was 35.6 hours and 75.1 hours, respectively.

Discussion: The total average cost of the ICD-10-CM implementation was calculated to be between $\$ 6,748$ to $\$ 9,564$ for a small medical practice and between $\$ 14,577$ to $\$ 23,062$ for a medium-sized medical practice. The results of this study suggest that for practices which used a set of free online resources, the eventual financial impact of the ICD-10-CM transition was less than predicted by the landmark Nachimson report for the American Medical Association (AMA), but greater than the study by Kravis et al (3M).

Keywords: World Health Organization; Education; Nachimson study; Merit-based incentive payment system

\section{*Corresponding author: Parth Desai \\ $\equiv$ desai_pn@med.mercer.edu}

MS-4, Mercer University School of Medicine, Macon, Georgia, USA.

Tel: +17064640772

Citation: Desai P, Eljazzar R (2018) PostImplementation Cost-Analysis of the ICD-10CM Transition on Small and Medium-Sized Medical Practices. J Health Med Econ Vol.4 No.1:4

Received: April 16, 2018; Accepted: May 16, 2018; Published: May 25, 2018

\section{Introduction}

On October 1, 2015, the United States made a major transition in its medical billing and coding system, by switching from ICD9-CM to ICD-10-CM. This transition resulted in an increase from 14,400 codes to around 144,00 possible diagnosis codes required to be documented by physicians for insurance reimbursement purposes [1]. ICD-10-CM is the most recent update to the International Statistical Classification of Diseases and Related
Health Problems (ICD) which was originally developed by the World Health Organization (WHO) and is used internationally for various epidemiological and healthcare reimbursement purposes [2]. ICD-10-CM will henceforth be referred to as simply, ICD-10.

The implementation of ICD-10 was a highly controversial issue in the U.S. healthcare industry, due to the potentially large financial impact that it was predicted to cause. The major concerns were the increased levels of specificity required for each case, the 
interruptions to clinician's work-flows due to the new language, the software readiness of smaller EMR vendors, and the paucity of available training and implementation resources [3]. In the years preceding the ICD-10 deadline, a tremendous amount of speculative research was conducted, aimed at estimating the potential financial burden of the ICD-10 coding system on hospitals and medical practices in America [4]. The results of these prior studies varied tremendously in their cost estimates and were debated widely in the industry. The purpose of our study is to assess the financial and productivity impact caused by the ICD-10 transition, based on post-implementation figures reported by a sub-set of small and medium-sized practices. This could then prove useful for determining the eventual impact of future changes to the healthcare reimbursement system, such as the Merit-Based Incentive Payment System (MIPS).

The largest and most widely publicized of the past ICD-10 costanalyses was a 2008 study funded by the American Medical Association (AMA) and conducted by Nachimson Advisors. The revised report, which was updated in 2014, found that the ICD-10 implementation would cost a typical small practice (3 physicians) between \$56,639 and \$226,105, and would cost a typical medium-sized practice (10 physicians) between $\$ 213,364$ and $\$ 824,735$ [5]. This was considered by the AMA to be a landmark study for healthcare policy in America and was defended by the organization's researchers amidst criticisms by a latter study in the field [6].

Three other major ICD-10 impact studies conducted in 2014 and early 2015, also intended to estimate the cost of the conversion for small and medium-sized medical practices. A 2014 study funded by $3 \mathrm{M}$ Health Information Systems and conducted by Kravis et al. estimated that the total conversion cost for a small practice consisting of three physicians and two impacted office personnel would range from $\$ 1,960-\$ 5,900$ [7]. The cost projections from this study varied significantly from the Nachimson study and were a source of controversy in the industry. The $3 \mathrm{M}$ study claimed that the cost estimated by the Nachimson study was highly inflated because it included costs of other healthcare initiatives not directly related to the ICD-10 conversion, and that ICD-10 resources were now available at lower costs since those previous estimates. The AMA however defended the validity of the Nachimson study and released a response claiming the $3 \mathrm{M}$ study "misstates the sources of the Nachimson Advisors study estimates" and "minimizes the efforts that practices must undertake to ensure a successful implementation [8]."

Another 2014 survey, conducted by the American Association of Professional Coders (AAPC), found that on average, the cost of ICD-10 would be $\$ 750$ per provider for clients of AAPC [9]. In early 2015, a fourth study surveyed 276 medical practices and found that the cost would range from $\$ 4,372$ for a practice with one physician, to $\$ 9,641$ for a practice with three physicians, to $\$ 11,028$ for a practice with six physicians [10]. These results, while greater than the estimates from the $3 \mathrm{M}$ and AAPC studies, are still much lower than the costs estimated by the Nachimson study.
Though the results of these latter three studies varied quite drastically from the Nachimson report, the commonality amongst them was that all were completed months to years prior to the actual ICD-10 implementation deadline. Our study provides a post-implementation cost analysis of the ICD-10 transition for a specific sub-set of practices which were registered clients of a non-profit providing free online software and training for ICD10. This data-analysis can then be used to compare to qualitative analyses on completed prior to the release of such free webbased resources to study the efficacy of such tools.

\section{Methods}

An online survey was distributed to a randomly selected sample of small and medium-sized medical practices in the United States by email. The population of interest for this study was specifically those medical practices consisting of between 1 to 8 physicians, which had completed the ICD-10 implementation using a set of free web-based resources. On 3 separate occasions (11/09/15, $11 / 19 / 15,12 / 01 / 15)$, the survey was emailed to exactly 6,000 randomly selected medical practices from the approximately 70,000 user database of the non-profit organization, ICD-10 Charts Inc. All 6,000 randomly selected practices were sent the survey invitation email, on each of the 3 dates, including those that had already completed the survey. In return for completing the survey, respondents were given a free ICD-10 coding tool. The survey consisted of seven questions designed to assess the financial and productivity impact of the ICD10 implementation on these various practices, as well as the practice demographics of the respondents. Survey respondents were asked to identify their practice's specialty, the state in four choices: 1-3 physicians, 4-8 physicians, 9-15 physicians, or $16+$ physicians. Next, respondents were asked to estimate the total number of hours their office staff spent on the ICD-10 implementation and the total number of hours their physicians spent on the implementation. The next question asked the total aggregate cost accrued for the implementation, followed by a specified cost breakdown of this total cost into the following categories: education, ICD-10 books/materials, ICD-10 readiness assessment, IT costs/software upgrades, testing costs, and other costs. Finally, respondents were asked if their reimbursement rates had increased, decreased, or stayed the same since the October 1, 2015 ICD-10 deadline. In order to confirm that each respondent represented a verified medical practice, respondents were required to provide their email address, which was crossreferenced with the organizational list of registered practices. This was also completed to ensure that each practice was only permitted one survey submission into the study.

\section{Results}

Of the 6,000 practices emailed the survey, a total of 512 practices from the pool of the non-profit clients responded, with a response rate of $8.5 \%$. Of these respondents, 58 survey responses were not included in the analysis because the size of these practices was larger than the population of interest (1-8 physician practices), and an additional 35 responses were removed due to incompletion of the survey. The remaining 419 responses were 
verified by email and analyzed anonymously to maintain privacy of the respondent's data. The 419 respondents represented medical practices from 43 different states, and across over 36 different medical specialties. 32\% (139) of the respondents were from primary-care practices, and 68\% (280) were from other specialty practices. Of the total 419 respondents, the majority $(82 \%)$, came from small practices, those consisting of 1-3 physicians, with only $18 \%$ coming from medium-sized practices (those consisting of 4-8 physicians).

Survey respondents provided the number of staff and physician hours spent on the ICD-10 transition, and the total cost of the ICD10 transition for their practice. Table 1 presents the average hours and the average total cost accrued by small and medium medical practices. The average hours spent on preparation was slightly more than double for medium practices (139 hours), compared to small practices (61.2 hours). Likewise, the average explicit cost of ICD-10 was also close to double for medium practices $(\$ 2,462)$ compared to small practices $(\$ 1,206)$, though it is important to note that this does not include the cost of productivity hours lost which will be considered later in this analysis.

These cost averages alone however, do not accurately represent the positively skewed nature of the distribution of explicit costs among these practices. The frequency distribution depicted in Figure 1 shows that the distribution is skewed to the left, with the highest frequency of respondents for both small and medium practices spending $\$ 0$ on their implementation (37\% and $49 \%$,

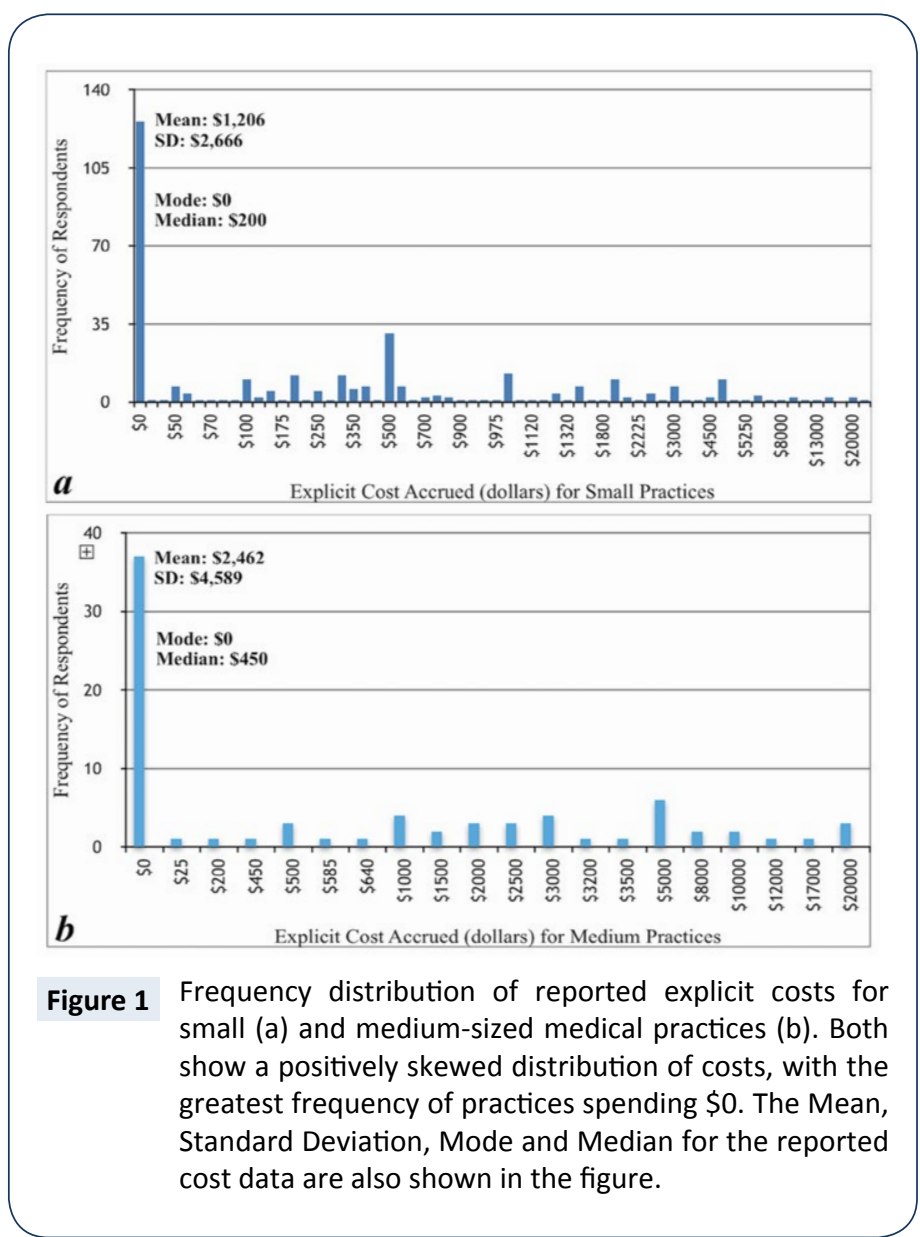

respectively). The mode of both sets of cost data was thus \$0, while the median for small practices was $\$ 200$, and $\$ 450$ for medium practices. In addition, $79 \%$ of small practices spent under the $\$ 1,206$ average, while $70 \%$ of medium practices spent under the \$2,462 average for their ICD-10 implementation. The standard deviation for the ICD-10 explicit cost data was $\$ 3,307$ for small practices and $\$ 4,658$ for medium practices. This suggests that significant variability was present in the amount of money spent on ICD-10 preparation among the medical practices of similar size.

It is important to note that the answers reported for the final survey question regarding if their practice's reimbursement rates had increased, decreased, or stayed the same since the ICD-10 deadline, was omitted from the analysis. The reason this question was omitted was because the survey was sent out prior to January 1, 2016 when the majority of practices would have begun seeing the respective denials and acceptances of claims submitted using ICD-10. Thus, it is likely that most respondents answered this question based on reimbursement rates for claims submitted using ICD-9, which is out scope for this study.

\section{Discussion}

The results of this study suggest that from our sample of 419 small and medium-sized medical practices who used a set of free online resources, the overall cost of the ICD-10 implementation was lower than cost estimates made by studies published prior to the implementation date. To make this comparison and accurately quantify the cumulative financial impact of the transition on the practices surveyed, it is beneficial to add the cost of work-hours spent on ICD-10 with the explicit cost average reported by our respondents.

The cost of productivity lost was determined by multiplying the hours spent on preparation by physicians and office staff, by the average hourly wage of physicians and office staff, respectively. The same average hourly salaries used in the Nachimson and Kravis studies, were also used for our calculations ( $\$ 43 /$ hour for office administrators and $\$ 81.73-\$ 194.71 /$ hour for physicians), to allow for comparison of our results to previous estimates (Table 2). By adding this average cost of time spent on ICD-10 with the average explicit cost reported by our respondents, the average total cost of the ICD-10 implementation was found to be between $\$ 6,748$ to $\$ 9,564$ for a small medical practice (1-3 physicians) and between $\$ 14,577$ to $\$ 23,062$ for a medium-sized medical practice.

As shown in Table 2, the average cumulative cost of ICD-10 for small practices responding to our survey was less than that estimated by the Nachimson report to the AMA, but greater than that estimated by the Kravis study, funded by $3 \mathrm{M}$. Specifically, our study found that the cumulative cost of the ICD-10 implementation for small practice respondents was between 5 to 33 times less than was estimated by the Nachimson report. One reason for the lower accrued costs reported by our respondents was that all of our respondents were users of a set of free ICD10 training and assessment resources, which did not exist at the time of the Nachimson report. This would have allowed the practices surveyed in this study to effectively implement ICD- 
Tables 1: The average number of staff hours, physician hours, and explicit cost spent on the ICD-10 implementation.

\begin{tabular}{|c|c|c|c|}
\hline & Average Total Staff Hours & Average Total Physician Hours & Average (Explicit) Cost Accrued \\
\hline Small Practice (1-3 physicians) & 61.2 hours & 35.6 hours & $\$ 1,206$ \\
\hline Medium Practice (4-8 physicians) & 139 hours & 75.1 hours & $\$ 2,462$
\end{tabular}

Table 2: Estimates from previous studies of the overall financial impact of the ICD-10 transition on small medical practices. The average cost of time was added to the average explicit cost to find the average total cost of ICD-10. The Buckholtz and Blanchette studies did not include cost of productivity hours spent, and thus only the average explicit costs estimated from these studies are shown. Note that the previous studies all included only 3 physician practices as small practices, while our study included practices ranging from 1 to 3 physicians in size.

\begin{tabular}{|c|c|c|c|c|c|c|c|}
\hline Study & Staff Hours & Staff Cost & $\begin{array}{l}\text { Physician } \\
\text { Hours }\end{array}$ & Physician Cost & $\begin{array}{l}\text { Average Cost } \\
\text { of Time }\end{array}$ & $\begin{array}{c}\text { Average Explicit } \\
\text { Cost }\end{array}$ & $\begin{array}{l}\text { Average Total Cost } \\
\text { of ICD-10 }\end{array}$ \\
\hline $\begin{array}{l}\text { Nachimson } 2014 \\
\text { (AMA) [8] }\end{array}$ & 200 hours & $\$ 8,600$ & 150 hours & $\$ 12,260-\$ 29,207$ & $\$ 31,812-\$ 63,897$ & $\$ 24,827-\$ 162,208$ & $\$ 56,639-\$ 226,105$ \\
\hline Kravis 2014 (3M) [7] & $16-32$ hours & $\$ 688-\$ 1,376$ & 9 hours & - & $\$ 1,520-\$ 3,500$ & $\$ 450-\$ 2,400$ & $\$ 1,970-\$ 5,900$ \\
\hline $\begin{array}{l}\text { Buckholtz } 2014 \\
\text { (AAPC) [9] }\end{array}$ & - & - & - & - & - & $\$ 2,250$ & - \\
\hline $\begin{array}{l}\text { Blanchette } 2015 \\
\text { (PAHCOM) [10] }\end{array}$ & - & - & - & - & - & $\$ 4,372-\$ 9,641$ & - \\
\hline Desai 2016 & 61.2 hours & $\$ 2,632$ & 35.6 hours & $\$ 2,910-\$ 6,932$ & $\$ 5,542-\$ 9,564$ & $\$ 1,206$ & $\$ 6,748-\$ 9,564$ \\
\hline
\end{tabular}

10 into their practice without any explicit costs on third-party ICD-10 vendors. Another reason, echoed by the majority of our respondents, was that a considerable portion of the transition process was completed by the respondent's EMR or practice management company. Based on the very high frequency (37\%) of small practices that reported $\$ 0$ in explicit costs for the ICD10 transition, it is clear that a large number of small practices in America either opted for free ICD-10 preparation options or relied heavily on their EMR vendors to complete the transition. One limitation with this analysis however, is that it did not include the additional cost of ICD-10 software upgrades which some EMR vendors incorporated directly into the annual fee to use the EMR. In these cases, the practice may not have reported any incurred cost, though they may have experienced an increase in their annual EMR expenses.

The Kravis study estimated lower costs for small practice ICD10 implementation than the incurred costs found in our study group (Table 2). The major reason for this difference was that the Kravis study estimates considered the hours spent on ICD10 training as the only cost of time lost, failing to include other hours associated with ICD-10 preparation. Based on the results of our study, the 16-32 hours estimated for staff and 9 hours estimates for physicians in the Kravis study were lower than the time required by our respondents for a successful transition. The actual hours that our respondent's practices reported spending on the transition were 2-4 times greater for office staff and roughly 4 times greater for physicians. The range of explicit costs estimated by Kravis $(\$ 450-\$ 2,400)$ however, overlap closely with our average explicit cost $(\$ 1,206)$.

An important difference in the methodology for our study compared to the Nachimson and Kravis reports was that both previous studies defined small practices as only those with exactly 3 physicians, while ours considered a range of 1-3 physicians to be a small practice. This range was intentionally chosen as such because it was determined that within our range, there was limited variation in explicit ICD-10 costs. This is supported by the large proportions of respondents from both small and medium-sized practices which reported $\$ 0$ in explicit costs for ICD-10. A limitation to using this method of grouping practice sizes is that impact cannot be assessed per physician and instead, is assessed per practice. The purpose of grouping by practice size however was because this was necessary to make an appropriate comparison to the Nachimson and Kravis studies, and because solo-practitioners constitute a larger proportion of the healthcare landscape than do 3 physician practices. The other remaining two studies on this topic, Buckholtz and Blanchette, are included in Table 2, but will not be compared further because these estimates did not consider the most significant portion of the overall financial impact of the ICD-10 transition, which was the cost of work-hours spent on the implementation. In addition, the results of medium-sized practices will not be compared to the previous studies, because so few of the studies included a specific estimate for practices with 4 to 8 physicians in their analyses.

Several factors contributed to the large differences in the previous estimates for the ICD-10 implementation costs, both compared to each other, and compared to our study. The key difference was the advent of free web-based resources which were utilized by all of the respondent's in this survey and had not existed even at the publication date of the previous analyses. Two other over-arching commonalties may also help account for the great deal of variation. The first commonality was that each of the previous studies was completed prior to the October 1 , 2015 ICD-10 deadline, making them more speculative in nature. This speculative nature afforded each research team the ability to create their own specific methodology from which to base the estimates-a fact that led to marked differences among the results. Each of the previous studies referenced in this paper conducted their research admirably in their own right, but the procedures and assumptions used to make their estimates varied too greatly from one another to accurately predict of the eventual impact of ICD-10, prior to the deadline. This underscores the importance of post-implementation cost-analysis studies such as 
ours, to better estimate determine the impact of industry-wide changes to healthcare documentation and payment systems.

The second commonality among the previous studies was that all were funded by national organizations having different agendas related to ICD-10. When considering the large variation among the cost estimates, it might be important to understand the specific ICD-10 agenda of each organization funding a particular study, because these potential sources of bias could have contributed to the differences in the methodology of the estimate, and thus might have contributed to determining the eventual results of the study. The AMA, the nation's largest physician advocacy organization, was for many years one of the largest opposition groups to the implementation of ICD-10 and a major contributor to the 3 multi-year delays of ICD-10 in the United States. As shown in the 2014 Physicians Foundation Biennial Survey, 75.3\% of the over 20,000 physician respondents stated that ICD-10 would unnecessarily complicate coding, and $50.1 \%$ stated that it would create a severe administrative problem [11]. It was thus perhaps not surprising that the AMA funded a study which greatly over-estimated the costs of the ICD-10 implementation, in order to prevent or delay the implementation of a change which they believed to be an unnecessary burden on physicians.

$3 \mathrm{M}$, on the other hand, is one of the nation's largest healthcare's IT companies and was invested in developing ICD10 implementation products to sell to medical practices. 3M had millions of dollars to gain by under-estimating the cost and impact of the ICD-10 transition, in order to prevent future delays and ensure the enactment of the ICD-10 system. This same trend of ICD-10 vendors under-estimating the financial impact of ICD10 was also revealed by analyzing another study by the American Association of Professional Coders (AAPC), in which the cost of the implementation in a small practice was found to be $\$ 750$ per provider. As described by healthcare IT expert and Editorin-Chief of Healthcare IT News, Tom Sullivan, "the case with just about all vendor-sponsored research" is that if the organization "stands to earn profits, if not customers," then the results of the research should be analyzed accordingly [12]. This demonstrates the role that potential monetization of any new healthcare documentation requirement has in dictating the eventual results of the cost-analysis research conducted and should be considered when analyzing future research conducted on the impact of the Merit-Based Incentive Payment System (MIPS).

When considering the potential for bias in organizational ICD10 research, it must also be mentioned that our study was conducted and written in-part by the founder of the non-profit organization, ICD-10 Charts Inc. ICD-10 Charts is a 501(c) 3 nonprofit organization that provides free ICD-10 education and implementation software to hospitals and medical practices. This relationship of the lead researcher to this non-profit organization could have certainly influenced the issues highlighted in this paper and therefore, must be considered appropriately during any analysis of the conclusions of this study. In addition, given the low $8.5 \%$ response rate for our survey, it is likely participation bias may have played a role due to respondents being more likely to have been frequent users of the non-profit resources.
The major limitation to the generalizability of this study to other small and medium-sized medical practices in the United States, is that all the practices surveyed were registered users of the free ICD-10 implementation resources created by ICD-10 Charts Inc. This allowed a large portion of the respondents to complete their entire implementation process, without spending additional money outside of the hours of productivity spent on the implementation. This likely contributed to the fact that such a large proportion of the total respondents (39\%) stated that they had incurred $\$ 0$ in explicit costs for the ICD-10, while others reported costs in the thousands for the same transition. This was further reflected by the large standard deviations $(\$ 3,307$ and $\$ 4,658$, respectively) for the cost data, which suggests that substantial variability existed in the overall cost of ICD-10 for different practices of similar size.

\section{Conclusions}

Based on the reported data of surveyed practices, the average total cost of the ICD-10 implementation was found to be between $\$ 6,748$ to $\$ 9,564$ for a small medical practice and between $\$ 14,577$ to $\$ 23,062$ for a medium-sized medical practice. The results of this study suggest that the financial impact of the ICD-10 transition on users of a set of free online resources was less than predicted by the landmark Nachimson report for the American Medical Association, but greater than the Kravis et al. study. Prior to the October 1, 2015 ICD-10 deadline, the results of every published ICD-10 cost-analysis estimated that there would be some explicit cost-however small or large associated with the ICD-10 transition. The findings of our study however, show that $39 \%$ of the total practices surveyed incurred $\$ 0$ in explicit costs.

Another important analysis not completed in this paper, that should be researched in future studies is the amount of stress that medical practitioners and their office personnel suffered during this difficult implementation process. This is important to address because the current studies available in the literature on this topic, focus primarily on the cost and work-hours spent on the implementation, but fail to address the significant amount of stress that accompanies any large-scale policy changes to the healthcare reimbursement system.

This information has implications for both the healthcare IT industry and in the future implementation of new health policy regulations in the United States. It highlights the large impact that non-profit, grant-supported enterprises can have in supporting small and medium-sized medical practices during health policy implementations in America. It also suggests the need for continued institutional support for organizations willing to develop and release free healthcare IT resources for future healthcare reimbursement systems, such as the Merit-Based Incentive Payment System (MIPS). To investigate the accuracy of our findings over time, we believe it is critical that this type of analysis be conducted again in the future, ideally with a larger and more balanced sample population. 


\section{References}

1 Manchikanti L, Kaye AD, Singh V, Boswell MV (2015) the Tragedy of the Implementation of ICD-10-CM as ICD-10: Is the Cart before the Horse or Is There a Tragic Paradox of Misinformation and Ignorance? Pain Physician 18: E485-495.

2 Hirsch JA, Nicola G, McGinty G (2016) ICD-10: History and context. Am J Neuroradiol 37: 596-599.

3 Watzlaf V, Alkarwi Z, Meyers S, Sheridan P (2015) Physicians' Outlook on ICD-10-CM/PCS and Its Effect on Their Practice. Perspect Health INF Manag.

4 Bowman S (2016) A Look Back on the ICD-10 Transition: Crisis Averted or Imaginary? J AHIMA 87:24-31.

5 Nachimson S, Hartley C. The Cost of Implementing ICD-10 for Physician Practices: Updating the 2008 Nachimson Advisors Study: A Report to the American Medical Association. US House of Representatives Document Repository.

6 Murphy K (2014) Nachimson Advisors Refutes ICD-10 Implementation Costs Claims. EHR Intelligence.
7 Kravis T, Belley S, Smith D, Averill R (2014) Cost of Converting Small Physician Offices to ICD-10 Much Lower than Previously Reported. Journal of AHIMA.

8 Nachimson Advisors. Nachimson Advisors Response to November 2014 Article in Journal of AHIMA "Cost of Converting Small Physician Offices to ICD-10 Much Lower than Previously Reported". Nachimson Advisors.

9 Buckholtz R (2014) Exclusive: ICD-10 Implementation-Where Do We Really Stand? ICD10 Monitor.

10 Blanchette K, Averill R, Bowman S (2015) Survey of ICD-10 Implementation Costs in Small Physician Offices. The PAHCOM Journal.

11 Survey of America's Physicians Practice Patterns \& Perspectives (2014) The Physicians Foundation.

12 Sullivan T (2015) Healthcare Providers Expect Major Financial Headaches from ICD-10, Survey Says. Healthcare Finance. 